\title{
Reactive Routing Protocol to Single Path with Load Balancing
}

\author{
Mekkakia Maaza Zoulikha \\ Department of Data processing, \\ University of Sciences and the \\ Technology of Oran \\ USTO, BP 1505 El M'Naouar, \\ Oran, Algeria
}

\author{
Ouali Sofiane \\ Department of Data processing, \\ University of Sciences and the \\ Technology of Oran \\ USTO, BP 1505 El M'Naouar, \\ Oran, Algeria
}

\author{
Sekrane Nassima \\ Department of Data processing, \\ University of Sciences and the \\ Technology of Oran \\ USTO, BP 1505 El M'Naouar, \\ Oran, Algeria
}

\begin{abstract}
Load balancing is a primary consideration in Adhoc networks, due to scarcity of resources. Equitable distribution of the load reduces consumption of network resources and provides homogeneous traffic characteristics in the network, such as load per node or the time from end-to-end incurred on each path.

In this paper we present an optimization of the reactive routing protocol AODV with load balancing. The goal is to provide a balanced distribution of traffic on different network nodes.
\end{abstract}

\section{Keywords}

Ad hoc, Load balancing, AODV, Routing.

\section{INTRODUCTION}

The problem of unbalanced load distribution in ad hoc networks is a limitation of routing protocols. While some nodes are not implicated in routing, others are strongly congested and carry the most traffic. With this inhomogeneous load balancing, nodes loaded quickly consume their limited material resources and show a high congestion, allowing a significant degradation of Ad hoc network performances.

Load balancing distributes traffic to a nodes set, in order to smooth the network load. That is to say, divide the total load to the various nodes of the network by sending data as nodes in a position to respond.

\section{LOAD BALANCING}

Load balancing aims to increase capacity and fault tolerance of networks. It is necessary to ensure a good load balancing on all ad hoc network paths. Load balancing to single path routing has been adopted in Ad hoc networks [1],[2],[3].

One of the first solutions provides a simple method, but very effective to achieve load balancing in an ad hoc network, is the mechanism WL "Workload-Based Adaptive Load Balancing" [4]. It was found that in the case of a reactive routing protocol (eg: AODV), any intermediate node that receives a RREQ message for a destination that has a path is required to send a reply RREP to participate in the routing traffic regardless of its condition. So the authors propose to offer freedom to a node to decide to respond to RREQ received messages or not, depending on its load. For this, each node defines thresholds for the queue and its load. This solution has the disadvantage of leaving the nodes are free to set their load thresholds. Just a node to set its threshold to the minimum value to reject automatically all the RREQ packet it receives.
This fault of mechanism does not really load balancing because the nodes using the protocol will be overloaded due to abuse of the "malicious" nodes.

Another solution is proposed protocol LBAR "Load-Balanced Ad hoc Routing" [5], is a reactive routing protocol. It was designed for demanding applications in terms of delay (latency). This protocol aims to provide less loaded paths, so that the packets are routed with less delay. The authors add a phase of calculation of the cost function to the first three phases: route discovery, route maintenance and management of local connectivity.

Based on the observation that nodes belonging to several active links, have a large load compared to those who have less, the authors propose a new cost function that takes into account this observation. Thus, for each route (path) $\mathrm{k}$, the metric considered is:

$\begin{aligned} \mathrm{C}_{\mathrm{k}} & =\sum\left(\mathrm{A}_{\mathrm{i}}+\mathrm{T}_{\mathrm{i}}\right) \\ & i<K\end{aligned}$

. Ai: The number of active links involving the node $\mathrm{i}$, an active link is a link where packets are being transmitted.

. Ti: The traffic interference of nodes $\mathrm{j}$ neighbors to $\mathrm{i}$. Ti can be written as follows::

$\mathrm{T}_{\mathrm{i}}=\sum \mathrm{A}_{\mathrm{i}}^{\mathrm{j}}$
$\quad j \in V(i)$

Thus, the proposed routing protocol aims to select routes to the least loaded nodes.

The LBAR protocol disadvantage of not considering in the cost function, that calculates, the number of hops between the source and destination. One can envisage scenarios where this protocol could push the source to choose a route (path) with a large number of nodes less loaded instead of a route with lightly loaded nodes.

\section{AODV WITH LOAD BALANCING}

The work of [6] shows that the load is maximum at the network center and the paths most used by the packets pass through the center nodes of the network. As a result, the network load is max at the center and decreases gradually as one moves away from it. The load balancing mechanism proposed in this section is based on this observation. So it would be sufficient to distribute the load, in a balanced manner on the network, to find a mechanism 
that pushes the load outside the center of the network, by selecting the least loaded paths.

The load balancing mechanism proposed is applied to the reactive protocol AODV [7]. We propose a new routing metric.

\section{PROPOSED A NEW METRIC FOR LOAD BALANCING}

In route discovery phase of AODV, if a source node wants to send data to a destination node, then the AODV routing protocol requires that the source broadcasts RREQ messages to the network. RREQ messages will be sent hop by hop until it reaches the destination (RFC 3561). This destination will issue a RREP response following the path by which succeeded the first RREQ. Then it ignores all other RREQ that are received by other paths. This rule will allow the destination node to consider only the shortest paths (the first arrived RREQ message has always taken the shortest path).

To choose less loaded paths, it is necessary that the destination node considers the RREQ messages backward. These messages announce paths with a hop count greater than or equal to the path announced by the first RREQ. We propose the following metric which allows the destination node selects the least loaded shortest path.

Min $1 / \mathrm{n} \sum$ nbr_of_flow(i)

$$
i € P
$$

- nbr_of_flow(i) denotes the number of flows traversing a node $\mathrm{i}$ and involved in the path $P$.

- $\mathrm{n}$ is the hops number.

This metric takes into account the load of path $\mathrm{P}$ in function of the hops number.

We also offer freedom, an intermediate node, deciding to participate in routing or not depending on its load. For this, we define a threshold for his load. This avoids overloading of node.

To allow the destination node to consider all the advertised paths, we eliminated the property of the intermediate node which is to respond to the source node by the RREP message, leave the decision only to destination node chooses the path to use. The rest of functioning of new protocol respects the recommendations of RFC 3561 [7].

Therefore, any node that receives a RREQ message, calculates the cost of the path announced and it will compare the cost of the existing path in its routing table, as follows:

If (request rq recently heard) then

For all entry in routing table

If (rt-> destination_address = rq-> source_address)then

$$
\begin{aligned}
& \mathrm{m} 1=\text { rq->rq_load } \\
& \mathrm{m} 2=\text { rt->rt_load } \\
& \text { If }(\mathrm{m} 1>\mathrm{m} 2) \text { then }
\end{aligned}
$$

Free paquet

\author{
Quit algorithm
}

End if

End if

End For

End if

Update corresponding entry

\section{PERFORMANCE EVLUATIONOF AODV WITH LOADBALANCING}

We have integrated our load balancing mechanism in an implementation of AODV in NS2 that we call AODV_B (AODV_Balanced).

In our simulations, the network topology is 20, 30, 50 and 60 nodes. The simulation time is 100 seconds.

Each node in the topology is configured as follows:

Table 1. Configuration parameters of a node

\begin{tabular}{|c|c|}
\hline Parameters & Values \\
\hline Type the MAC layer & IEEE 802.11 \\
\hline Buffer size of nodes & 50 packets \\
\hline Radio propagation model & Two Ray Ground \\
\hline Physical layer & bandwidth $2 \mathrm{Mb} / \mathrm{s}$ \\
\hline
\end{tabular}

For the four topologies, we generate the same number of traffic (5 CBR flows).

Table 2. Traffic parameters

\begin{tabular}{|c|c|}
\hline Parameters & Values \\
\hline Type of traffic & CBR \\
\hline Number of nodes & $(20,30,50,60)$ \\
\hline Sending frequency & 2 packets/s \\
\hline Packet size & 512 bytes \\
\hline
\end{tabular}

Fig 1 shows the simulation results of a topology of 20 nodes, 5 traffic sources and simulation time of 100 s, using the protocols AODV and AODV_Balanced (AODV_B).

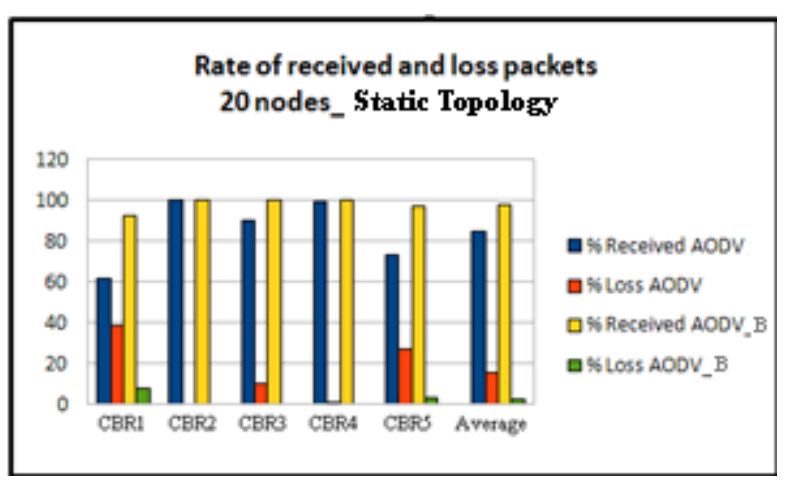




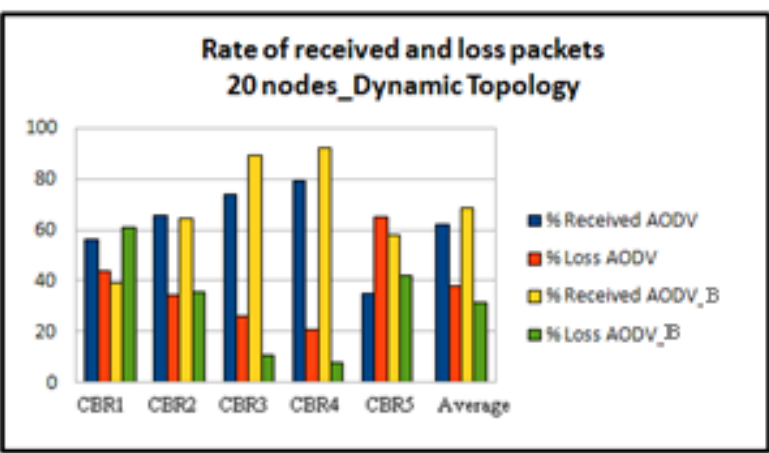

Fig 1: Comparison between AODV and AODV_B
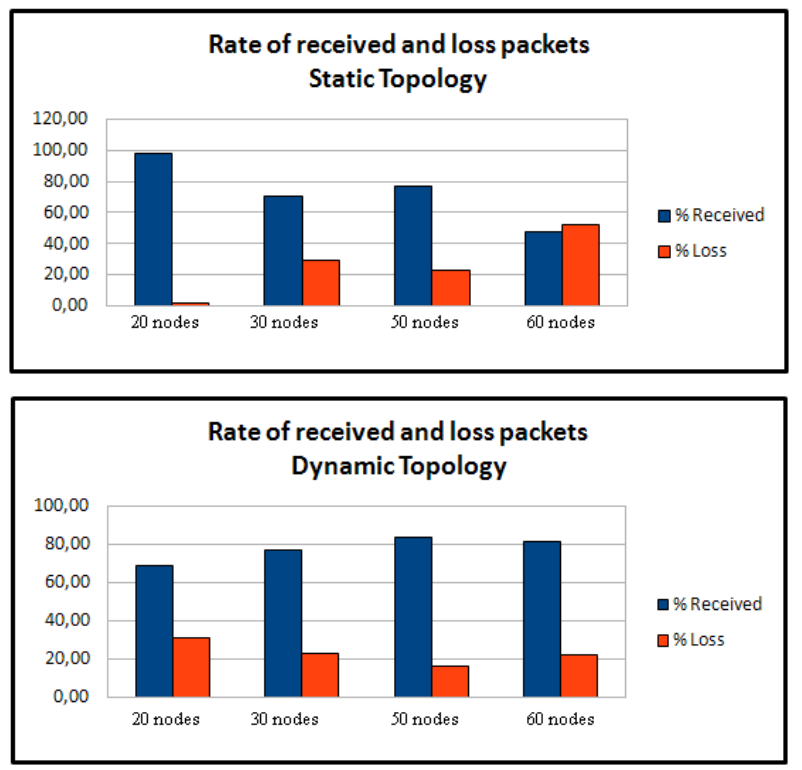

Fig 2: AODV_B as a function of the density

AODV protocol is known to have high reliability compared to existing routing protocols. However, as soon as the load increases, reliability decreases significantly. For the scenarios we considered, we found the packet delivery ratios around $84 \%$ for static topology, and $62 \%$ for dynamic topology. Through the mechanism of load balancing (AODV_B), the reliability has been substantially increased to reach values $97 \%$ (static topology) and 68\% (dynamic topology).

This improvement is due to the choice of paths less loaded. If the packets through lightly loaded nodes, they are more likely to find queues unsaturated and therefore have less probability of rejection.

In terms of mobility, AODV_B showed more robust when the network density increases, because the mechanism of load balancing traffic pushes outside of the most charged nodes, whenever there is a path less charged, avoiding network congestion.

In terms of density, we find a better load balancing when nodes are mobile, this is because of existence of a multitude of paths less charged (or not loaded), allowing the nodes to avoid openpaths use.

\section{CONCLUSIONS}

The traffic concentration in the center of network is due to the use of the routing algorithm shortest path, because such a path passes through the central nodes. The use of load balancing mechanism reduces the load center of network and increases the involvement of edge nodes in routing. The load balancing mechanism proposed is based on the selection of least loaded shortest path, so the paths are furthest from the center of network, reducing the load of central nodes.

\section{ACKNOWLEDGMENTS}

This work is part of the CAPRAH project 08/U311/4966, supported by the Algerian Ministry of Higher Education and Scientific Research.

\section{REFERENCES}

[1] Wenbin Jiang Zhaojing Li Chunqiang Zeng Hai Jin, 2009, Load Balancing Routing Algorithm for Ad Hoc Networks, 5th International Conference on Mobile Ad-hoc and Sensor Networks (MSN), 2009

[2] Toh Chai Keong, Le Anh-Ngoc and Cho You-Ze,2009, Load Balanced Routing Protocols for Ad Hoc Mobile Wireless Networks, IEEE Communications Magazine, Institute of Electrical and Electronics Engineers Inc., Vol. 47, No. 8, pp. 78-84.

[3] Y. Yuan, H. Chen, and M. Jia. 2005. An adaptative load balancing approach for Ad Hoc networks. Wireless Communications, Networking and Mobile Computing international Conference, Vol. 2.

[4] Y.J.Lee, G.F.Riley. 2005. A workload-based adaptative load-balancing technique for mobile Ad Hoc network. IEEE Wireless Communications and Networking Conference (WCNC'2005)

[5] P. Pham and S. Perreau. 2002. Multi-path routing protocol with load balancing policy in mobile ad hoc network. in IEEE MWCN'02.

[6] P.Pham, S. Perreau. 2003. Performance analysis of reactive shortest path and multi-path routing mechanisms with load balance. IEEE Conference on Computer Communications INFOCOM'03.

[7] C. Perkins, S. Das. 2003. Ad hoc On-Demand Distance Vector (AODV) Routing. RFC 3561 IETF, URL: http://www.ietf.org/rfc/rfc3561.txt 